\title{
The Roadmap to the POEMMA mission
}

\section{A. V. Olinto ${ }^{a, *}$ and J. F. Krizmanic ${ }^{b}$ on behalf of the POEMMA Collaboration} (a complete list of authors can be found at the end of the proceedings)

${ }^{a}$ Department of Astronomy \& Astrophysics, University of Chicago, Chicago, IL 60637, USA

${ }^{b}$ Center for Space Science \& Technology, University of Maryland, Baltimore County, Baltimore, and NASA Goddard Space Flight Center, Greenbelt, MD, USA

E-mail: aolinto@uchicago.edu

The Probe Of Extreme Multi-Messenger Astrophysics (POEMMA) is designed to observe ultrahigh-energy cosmic rays (UHECRs) and cosmic neutrinos from space with sensitivity over the full celestial sky. Developed as a NASA Astrophysics Probe-class mission, POEMMA consists of two identical telescopes orbiting the Earth in a loose formation designed to observe extensive air showers (EAS) via air fluorescence and Cherenkov emissions. UHECRs and UHE neutrinos above $20 \mathrm{EeV}$ are observed with the stereo fluorescence technique, while tau neutrinos above 20 $\mathrm{PeV}$ are observed via the optical Cherenkov signals produced by up-going EAS generated by the decay of Earth-emerging tau-leptons. The POEMMA satellites are designed to quickly re-orientate to follow up transient cosmic neutrino candidate sources and obtain unparalleled neutrino flux sensitivity. Both observation techniques and the instrument design are being validated by current and upcoming missions, such as Mini-EUSO and EUSO-SPB as part of the JEM-EUSO program, and the Terzina instrument onboard the NUSES SmallSat mission. We discuss the POEMMA science performance and the current roadmap to the POEMMA mission.

$37^{\text {th }}$ International Cosmic Ray Conference (ICRC 2021)

July 12th - 23rd, 2021

Online - Berlin, Germany

\footnotetext{
*Presenter
} 


\section{Introduction}

The Probe Of Extreme Multi-Messenger Astrophysics (POEMMA) is one of the probe mission proposals selected for a conceptual study funded by NASA in preparation for the Astro2020 decadal survey. The POEMMA design optimizes the observation of extensive airshowers (EASs) from space by observing the ultraviolet emission from atmospheric fluorescence and the Cherenkov emission from upward going EASs. The stereo fluorescence technique enables precise observations of ultrahigh-energy cosmic rays (UHECRs) and ultrahigh-energy cosmic neutrinos above about 20 $\mathrm{EeV}$. The Cherenkov technique enables the observation of tau-neutrinos from upward going EASs produced by tau-lepton decay generated by tau neutrinos that traverse the Earth and convert into tau-leptons. The Cherenkov technique is optimized for following targets-of-opportunity (ToO) transient astrophysical events that may emit neutrinos above $20 \mathrm{PeV}$. A detailed description of the POEMMA instrument, mission, and science can be found in Ref. [1] and references therein.

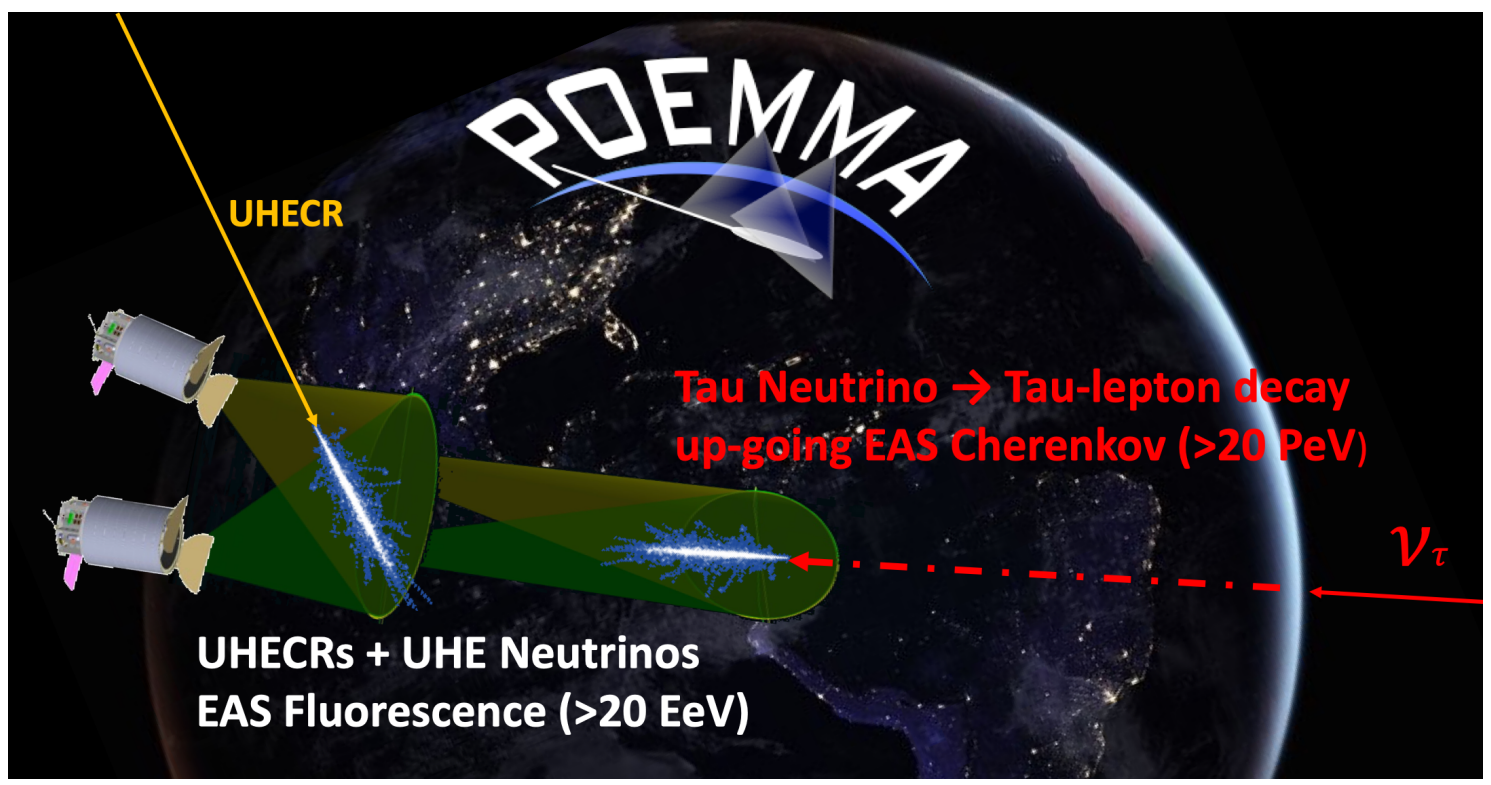

Figure 1: POEMMA Observation Modes: UHECRs and UHE neutrinos with energies above $20 \mathrm{EeV}$ are observed by the ultraviolet emission from atmospheric fluorescence produced by the EASs generated by these particles as they interact in the atmosphere. A target-of-opportunity program to observe tau neutrinos with energies above $20 \mathrm{PeV}$ involves the observation of Cherenkov emission from upward going EAS produced by tau-lepton decay generated by tau neutrinos that traverse the Earth.

The main questions POEMMA is designed to answer are the origin of ultrahigh-energy cosmic rays (UHECRs) and the origin of astrophysical neutrinos. POEMMA will address these questions by measuring the spectrum, composition, and anisotropies over the full sky of UHECRs with energies above $20 \mathrm{EeV}$, and observing astrophysical neutrinos above $20 \mathrm{PeV}$ through a target-of-opportunity (ToO) program designed to study neutrino rich multi-messenger electromagnetic and gravitational wave sources. POEMMA will study the physics and astrophysics at energies well above groundbased accelerators, where signatures of new interactions or dark matter may be present (see, e.g., secret neutrino interactions [1,2], supermassive dark matter [1, 3], and macroscopic dark matter $[1,4])$. 




\begin{tabular}{|c|c|c|c|c|}
\hline \multicolumn{3}{|c|}{ Photometer Components } & \multicolumn{2}{|l|}{ Spacecraft } \\
\hline Optics & Schmidt & $45^{\circ}$ full FoV & Slew rate & $90^{\circ}$ in $8 \min$ \\
\hline & Primary Mirror & $4 \mathrm{~m}$ diam. & Pointing Res. & $0.1^{\circ}$ \\
\hline & Corrector Lens & $3.3 \mathrm{~m}$ diam. & Pointing Know. & $0.01^{\circ}$ \\
\hline & Focal Surface & $1.6 \mathrm{~m}$ diam. & Clock synch. & $10 \mathrm{nsec}$ \\
\hline & Pixel Size & $3 \times 3 \mathrm{~mm}^{2}$ & Data Storage & 7 days \\
\hline & Pixel FoV & $0.084^{\circ}$ & Communication & S-band \\
\hline PFC & MAPMT $(1 \mu \mathrm{s})$ & 126,720 pixels & Wet Mass & $3,450 \mathrm{~kg}$ \\
\hline PCC & $\operatorname{SiPM}(20 \mathrm{~ns})$ & 15,360 pixels & Total Power & $880 \mathrm{~W}$ \\
\hline \multicolumn{3}{|c|}{ Photometer (One) } & Mission & (2 Observatories) \\
\hline & Mass & $1,550 \mathrm{~kg}$ & Lifetime & 3 year ( 5 year goal) \\
\hline & Power & $590 \mathrm{~W}$ & Orbit & $525 \mathrm{~km}, 28.5^{\circ} \mathrm{Inc}$ \\
\hline & Data & $<1 \mathrm{~GB} /$ day & Orbit Period & $95 \mathrm{~min}$ \\
\hline & & & \multicolumn{2}{|c|}{ Observatory Sep. $\sim 25-1000+\mathrm{km}$} \\
\hline
\end{tabular}

Each Observatory $=$ Photometer + Spacecraft; POEMMA Mission $=2$ Observatories

Figure 2: POEMMA telescopes: The POEMMA observatory comprises of two identical telescopes with a 45 degrees field-of-view (FoV) Schmidt optics provided by a 4-meter mirror, a hybrid focal surface, and a corrector lens designed to reach an optical collection of over $6 \mathrm{~m}^{2}$. Figure and table adapted from Ref. [1].

The orbit and optical design of POEMMA enables instantaneous observations of colossal volumes of the atmosphere with projection areas on the ground from $10^{5} \mathrm{~km}^{2}$ in Nadir pointing to $10^{7} \mathrm{~km}^{2}$ when pointed at the limb of the Earth. The mission involves deploying two telescopes delivered by a dual manifest on an Atlas $\mathrm{V}$ to a $525 \mathrm{~km}$ altitude orbit with 28.5 degree inclination. The orbital period is $95 \mathrm{~min}$ and the mission goal is 5 years. Each telescope has a very wide 45 degrees field-of-view (FoV) optics provided by a 4-meter mirror, a hybrid focal surface, and a creative corrector lens that gives an optical collection area of over $6 \mathrm{~m}^{2}$. The hybrid focal surface has 126,720 pixels of MAPMTs for fluorescence observations with $1 \mu$ s sampling and 15,360 pixels of SiPMs for Cherenkov detection with 20 ns sampling. Both both types of cameras will be flown in 2023 on the Extreme Universe Space Observatory on a Super Pressure Balloon (EUSO-SPB2) [5].



Figure 3: The POEMMA Hybrid Focal Surface was designed with 126,720 pixels of MAPMTs for fluorescence observations with $1 \mu$ s sampling and 15,360 pixels of SiPMs for Cherenkov detection with $20 \mathrm{~ns}$ sampling. EUSO-SPB2 has both camera types.

POEMMA will reach unprecedented statistics above $20 \mathrm{EeV}$ to study the sources of UHECRs 

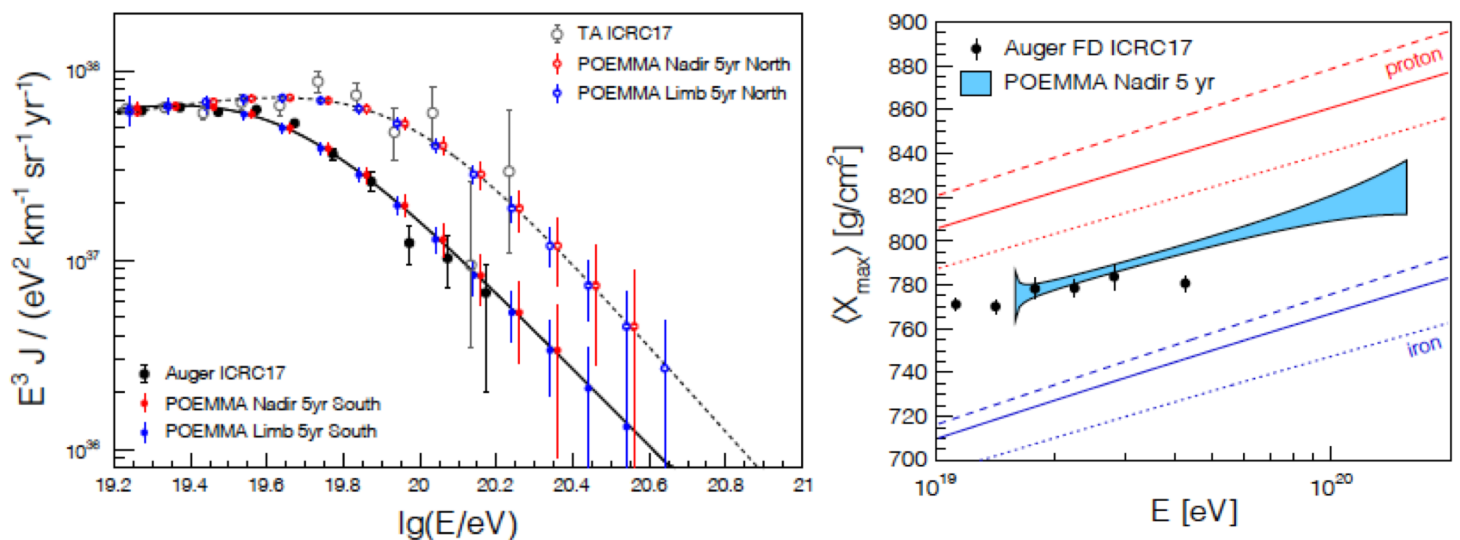

Figure 4: POEMMA UHECR Science: Left: Simulated POEMMA spectra compared with Auger 2020 (black dots and solid line) from Ref. [6] and the TA 2019 (black open circles and dotted line) from Ref. [7] for both POEMMA stereo Nadir observations (red) and POEMMA Limb observations (blue). Figure adapted from Ref. [1, 8]. Right: Capability of POEMMA to measure $\left\langle X_{\max }\right\rangle$ for composition studies at UHEs. The width of the blue band illustrates the expected statistical uncertainties in five years of POEMMA-Stereo (nadir) operations given the number of events per 0.1 in the logarithm of energy, the $X_{\max }$ resolution and efficiency for $\theta<70^{\circ}$, and the intrinsic shower-to-shower fluctuations of $40 \mathrm{~g} / \mathrm{cm}^{2}$. The black dots are fluorescence data from Auger ICRC 2019 [9] and the blue bands are from Ref. [1, 8].

with energy resolution of $17 \%$, angular resolution of about 1 degree, and $X_{\max }$ resolution below $30 \%$ all at $100 \mathrm{EeV}$ [8]. This will extend our UHECR observations of spectrum and composition above $100 \mathrm{EeV}$ with a complete sky coverage at energies where anisotropies should become sharper and sources can be identified (see, e.g., $[1,10,11]$ ).
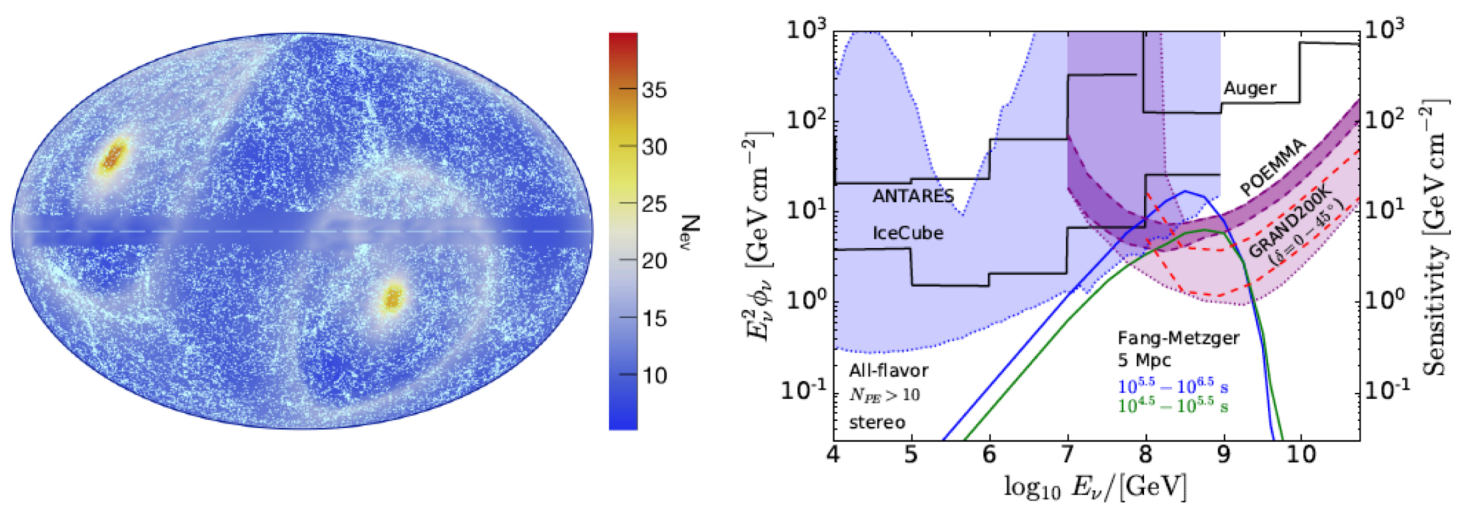

Figure 5: POEMMA Neutrino Science: Left: Sky plot of the expected number of neutrino events with POEMMA as a function of galactic coordinates for the Fang and Metzger [12] binary neutron star merger model, placing the source at $10 \mathrm{Mpc}$ Refs. [1, 13]. Right: ToO sensitivities to a long burst shown by the magenta band as in Refs. [1, 13]. Also shown are the IceCube all-flavor upper limits (solid histogram) from the GW170817 neutrino search [14]. The red dashed curves represent the projected sensitivity of GRAND200k [15], and models from Fang and Metzger [12] of the all-flavor neutrino fluence produced $10^{5.5}-10^{6.5} \mathrm{~s}$ and $10^{4.5}-10^{5.5} \mathrm{~s}$ after a binary neutron star merger event occurring at a distance of $10 \mathrm{Mpc}$. Figures adapted from $[1,13]$

POEMMA will also search for UHE neutrinos with fluorescence and have a ToO program 
to observe neutrinos from multi-messenger transient events. POEMMA will observe Cherenkov emission from EAS produced as tau neutrinos cross the Earth and produce tau leptons that decay on their way out of the Earth's surface producing up-going showers [16]. The ToO program will cover tau-neutrino energies above $20 \mathrm{PeV}$, with full sky coverage, and great sensitivity to many neutrino rich astrophysical transients, such as neutron-star binary coalescence, tidal disruption events, and soft gamma ray bursts [17].

The roadmap to the POEMMA mission starts with previous work for the OWL (Orbiting Wide-field Light-collectors) [18, 19] design, the JEM-EUSO (Joint Experiment Missions for the Extreme Universe Space Observatory) [20] program, the CHANT (CHerenkov Astrophysical Neutrino Telescope) concept [21], and the sub-orbital payloads EUSO-SPB1 (Extreme Universe Space Observatory on a Super Pressure Balloon 1) [22] and EUSO-SPB2 [5]. The OWL designed introduced the stereo vision of two free-flyers with large reflecting optics similar to POEMMA. The JEM-EUSO program developed a number of projects built with the MAPMT cameras like the one proposed for the POEMMA fluorescence system. Currently under construction and scheduled to fly in 2023, EUSO-SPB2 has both a fluorescence telescope pointing Nadir and a Cherenkov telescope pointing towards the limb of the Earth [5, 16, 23]. EUSO-SPB2 will establish both detection techniques for POEMMA. In addition to EUSO-SPB2, the NUSES SmallSat mission is planning to carry the Terzina instrument designed to establish the Cherenkov emission of cosmic rays above the limb [23]. On the ground, the Trinity [24] observatory will also be testing the POEMMA Cherenkov detection technique.



Figure 6: EUSO-SPB2 will test both detection methods of POEMMA. Planned for a 2023 flight, EUSOSPB2 will observe UHECRs from above with the fluorescence telescope and cosmic rays above the limb with the Cherenkov camera [5].

\section{Conclusion}

In conclusion, POEMMA will use the Earth's Atmosphere as a gigantic particle observatory to discover the origin of the highest energy cosmic rays $(\mathrm{E}>100 \mathrm{EeV})[1,11]$ and to observe very high energy $(\mathrm{E}>20 \mathrm{PeV})$ neutrino emission from astrophysical transient events $[1,17]$. These studies 
will bring new insight onto the most extremely energetic environments in the present universe enabling the study of new astrophysical and physical phenomena.

Acknowledgment: The conceptual design of POEMMA was supported by NASA Probe Mission Concept Study grant NNX17AJ82G for the 2020 Decadal Survey Planning. Contributors to this work were supported in part by NASA awards 16-APROBES16-0023, 17-APRA17-0066, NNX17AJ82G, NNX13AH54G, 80NSSC18K0246, 80NSSC18K0473, 80NSSC19K0626, and 80NSSC18K0464. The design for the POEMMA observatory, including two telescopes (instrument and spacecraft) and the mission concepts, was refined at the Integrated Design Center (IDC) of the Goddard Space Flight Center (GSFC). The POEMMA instrument was developed by the POEMMA collaboration with input from the IDC Instrument Design Laboratory (IDL). The POEMMA spacecraft and mission concepts were developed at the IDC Mission Design Laboratory (MDL). MB is supported by the VILLUM FondEN under project no. 29388. The authors from University of Torino acknowledge support from Compagnia di San Paolo within the project ex-post-2018. The Czech authors are supported by the Ministry of Education, Youth and Sports of the Czech Republic. GMT is supported by PAPIIT IN111621/DGAPA/UNAM.

\section{References}

[1] A.V. Olinto et al., The POEMMA (Probe of Extreme Multi-Messenger Astrophysics) observatory, Journal of Cosmology and Astroparticle Physics 2021 (2021) 007.

[2] M. Bustamante, New interactions of ultra-high-energy neutrinos: end-to-end forecasts for upcoming neutrino telescopes, in Proceedings, 37th International Cosmic Ray Conference (ICRC 2021): Berlin, Germany, July 12-23, 2021, vol. ICRC2021, p. 1222, 2021.

[3] Guépin et al., Probing the properties of superheavy dark matter with ultra-high energy neutrino experiments, in Proceedings, 37th International Cosmic Ray Conference (ICRC 2021): Berlin, Germany, July 12-23, 2021, vol. ICRC2021, p. 551, 2021.

[4] T. Paul et al., Model independent search for macroscopic dark matter with EUSO-SPB2, in Proceedings, 37th International Cosmic Ray Conference (ICRC 2021): Berlin, Germany, July 12-23, 2021, vol. ICRC2021, p. 519, 2021.

[5] J. Eser et al., Science and mission status of EUSO-SPB2, in Proceedings, 37th International Cosmic Ray Conference (ICRC 2021): Berlin, Germany, July 12-23, 2021, vol. ICRC2021, p. 404, 2021.

[6] A. Aab et al. [Pierre Auger Collaboration], Features of the Energy Spectrum of Cosmic Rays above $2.510^{18} \mathrm{eV}$ Using the Pierre Auger Observatory, Phys. Rev. Lett.,125,121106 (2020) [arXiv:2008.06488 [astro-ph.HE]].

[7] D. Ivanov [Telescope Array Collaboration] Energy Spectrum Measured by the Telescope Array Experiment, PoS ICRC 2019, 298 (2019).

[8] L. A. Anchordoqui, D. R. Bergman, M. E. Bertaina, F. Fenu, J. F. Krizmanic, A. Liberatore, A. V. Olinto, M. H. Reno, F. Sarazin, K. Shinozaki, J. F. Soriano, R. Ulrich, M. Unger, T. M. Venters and L. Wiencke, Performance and science reach of the Probe of Extreme Multi-Messenger Astrophysics for ultrahigh-energy particles, Phys. Rev. D 101, no.2, 023012 (2020) doi:10.1103/PhysRevD.101.023012 [arXiv:1907.03694 [astro-ph.HE]].

[9] A. Yushkov et al. [Pierre Auger Collaboration], Mass Composition of Cosmic Rays with Energies above $10^{17.2} \mathrm{eV}$ from the Hybrid Data of the Pierre Auger Observatory, PoS, ICRC2019, 482, 2020.

[10] R. Alves Batista, J. Biteau, M. Bustamante, K. Dolag, R. Engel, K. Fang et al., Open questions in cosmic-ray research at ultrahigh energies, Frontiers in Astronomy and Space Sciences 6 (2019).

[11] T. Venters et al., Prospects for Cross-correlations of UHECR Events with Astrophysical Sources with Upcoming Space-based Experiments, in Proceedings, 37th International Cosmic Ray Conference (ICRC 2021): Berlin, Germany, July 12-23, 2021, vol. ICRC2021, p. 419, 2021. 
[12] K. Fang and B.D. Metzger, High-energy neutrinos from millisecond magnetars formed from the merger of binary neutron stars, The Astrophysical Journal 849 (2017) 153.

[13] T. M. Venters, M. H. Reno, J. F. Krizmanic, L. A. Anchordoqui, C. Guépin and A. V. Olinto, POEMMA's target of opportunity sensitivity to cosmic neutrino transient sources, Phys. Rev. D 102, 123013 (2020), [arXiv:1906.07209 [astro-ph.HE]].

[14] A. Albert et al. [ANTARES and IceCube and Pierre Auger and LIGO Scientific and Virgo Collaborations], Search for High-energy Neutrinos from Binary Neutron Star Merger GW170817 with ANTARES, IceCube, and the Pierre Auger Observatory, Astrophys. J. 850, no. 2, L35 (2017) doi:10.3847/2041-8213/aa9aed [arXiv:1710.05839 [astro-ph.HE]].

[15] J. Álvarez-Muñiz et al. [GRAND], The Giant Radio Array for Neutrino Detection (GRAND): Science and Design, Sci. China Phys. Mech. Astron. 63 (2020) no.1, 219501 doi:10.1007/s11433-018-9385-7 [arXiv:1810.09994 [astroph.HE]].

[16] M.H. Reno et al., EAS Optical Cherenkov signatures of tau neutrinos for space and suborbital detectors in the Proceedings, 37th International Cosmic Ray Conference (ICRC 2021): Berlin, Germany, July 12-23, 2021, vol. ICRC2021, p. 1201, 2021.

[17] T. Venters et al., Astrophysical Implications of Neutrino Target-of-Opportunity Observations with Space-based and Suborbital Optical Cherenkov Detectors, in the Proceedings, 37th International Cosmic Ray Conference (ICRC 2021): Berlin, Germany, July 12-23, 2021, vol. ICRC2021, p. 977, 2021.

[18] F. W. Stecker, J. F. Krizmanic, L. M. Barbier, E. Loh, J. W. Mitchell, P. Sokolsky and R. E. Streitmatter, Observing the ultrahigh-energy universe with OWL eyes, Nucl. Phys. Proc. Suppl. 136C, 433 (2004) doi:10.1016/j.nuclphysbps.2004.10.027 [astro-ph/0408162].

[19] J. F. Krizmanic and J. W. Mitchell, The Potential of Spaced-based High-Energy Neutrino Measurements via the Airshower Cherenkov Signal, doi:10.7529/ICRC2011/V04/1331 [arXiv:1109.6544 [astro-ph.HE]].

[20] J. H. Adams et al. [JEM-EUSO Collaboration], An evaluation of the exposure in nadir observation of the JEM-EUSO mission, Astropart. Phys. 44, 76 (2013) doi:10.1016/j.astropartphys.2013.01.008 [arXiv:1305.2478 [astro-ph.HE]].

[21] A. Neronov, D. V. Semikoz, L. A. Anchordoqui, J. Adams and A. V. Olinto, Sensitivity of a proposed space-based Cherenkov astrophysical-neutrino telescope, Phys. Rev. D 95, no. 2, 023004 (2017) doi:10.1103/PhysRevD.95.023004 [arXiv:1606.03629 [astro-ph.IM]].

[22] L. Wiencke, A. Olinto et al., EUSO-SPB1 Mission and Science, in the Proceedings, 35th International Cosmic Ray Conference (ICRC 2017): Busan, South Korea, July 10-20, 2017, vol. ICRC2017, p. 1097, 2017.

[23] A. Cummings, R. Aloisio, J. Eser and J. Krizmanic, Detection of Above the Limb Cosmic Rays in the Optical Cherenkov Regime Using Sub-Orbital and Orbital Instruments, in the Proceedings, 37th International Cosmic Ray Conference (ICRC 2021): Berlin, Germany, July 12-23, 2021, vol. ICRC2021, p. 437, 2021.

[24] A. Brown et al., Trinity: an imaging air Cherenkov telescope to search for Ultra-High-Energy neutrinos in Proceedings, 37th International Cosmic Ray Conference (ICRC 2021): Berlin, Germany, July 12-23, 2021, vol. ICRC2021, p. 1179, 2021. 


\section{Full Authors List: POEMMA}

J. H. Adams, ${ }^{1}$ R. Aloisio, ${ }^{2}$ L. A. Anchordoqui, ${ }^{3}$ A. Anzalone,${ }^{4,5}$ M. Bagheri, ${ }^{6}$ D. Barghini, ${ }^{7}$ M. Battisti, ${ }^{7}$ D. R. Bergman, ${ }^{8}$ M. E. Bertaina, ${ }^{7}$ P. F. Bertone, ${ }^{9}$ F. Bisconti, ${ }^{10}$ M. Bustamante, ${ }^{11}$ F. Cafagna, ${ }^{12}$ R. Caruso, ${ }^{13,5}$ M. Casolino, ${ }^{14,15}$ K. Černý, ${ }^{16}$ M. J. Christl, ${ }^{9}$ A. L. Cummings, ${ }^{2}$ I. De Mitri, ${ }^{2}$ R. Diesing, ${ }^{17}$ R. Engel, ${ }^{18}$ J. Eser, ${ }^{17}$ K. Fang, ${ }^{19}$ F. Fenu, ${ }^{7}$ G. Filippatos, ${ }^{20}$ E. Gazda, ${ }^{6}$ C. Guepin, ${ }^{21}$ A. Haungs, ${ }^{18}$ E. A. Hays ${ }^{22}$ E. G. Judd, ${ }^{23}$ P. Klimov, ${ }^{24}$ J. Krizmanic, ${ }^{22,25}$ V. Kungel, ${ }^{20}$ E. Kuznetsov, ${ }^{1} \check{S S}_{\text {. Mackovjak }}{ }^{26}$ D. Mandát, ${ }^{27}$ L. Marcelli, ${ }^{15}$ J. McEnery, ${ }^{22}$ G. Medina-Tanco, ${ }^{28}$ K.D. Merenda, ${ }^{20}$ S. S. Meyer, ${ }^{17}$ J. W. Mitchell, ${ }^{22}$ H. Miyamoto, ${ }^{7}$ J. M. Nachtman, ${ }^{29}$ A. Neronov, ${ }^{30}$ F. Oikonomou, ${ }^{31}$ A. V. Olinto, ${ }^{17}$ Y. Onel, ${ }^{29}$ G. Osteria, ${ }^{32}$ A. N. Otte, ${ }^{6}$ E. Parizot, ${ }^{33}$ T. Paul, ${ }^{3}$ M. Pech, ${ }^{16}$ J. S. Perkins, ${ }^{22}$ P. Picozza, ${ }^{15,34}$ L.W. Piotrowski, ${ }^{35}$ Z. Plebaniak, ${ }^{7}$ G. Prévôt, ${ }^{33}$ P. Reardon, ${ }^{1}$ M. H. Reno, ${ }^{29}$ M. Ricci, ${ }^{36}$ O. Romero Matamala, ${ }^{6}$ F. Sarazin, ${ }^{20}$ P. Schovánek, ${ }^{27}$ V. Scotti, ${ }^{32,37}$ K. Shinozaki, ${ }^{38}$ J. F. Soriano, ${ }^{3}$ F. Stecker, ${ }^{22}$ Y. Takizawa, ${ }^{14}$ R. Ulrich, ${ }^{18}$ M. Unger, ${ }^{18}$ T. M. Venters, ${ }^{22}$ L. Wiencke, ${ }^{20}$ D. Winn, ${ }^{39}$ R. M. Young, ${ }^{9}$ M. Zotov ${ }^{24}$.

${ }^{1}$ University of Alabama in Huntsville, Huntsville, AL, USA; ${ }^{2}$ Gran Sasso Science Institute, L'Aquila, Italy; ${ }^{3}$ City University of New York, Lehman College, NY, USA; ${ }^{4}$ Istituto Nazionale di Astrofisica INAF-IASF, Palermo, Italy; ${ }^{5}$ Istituto Nazionale di Fisica Nucleare, Catania, Italy; ${ }^{6}$ Georgia Institute of Technology, Atlanta, GA, USA; ${ }^{7}$ Universita' di Torino, Torino, Italy; ${ }^{8}$ University of Utah, Salt Lake City, Utah, USA $;{ }^{9}$ NASA Marshall Space Flight Center, Huntsville, AL, USA; ${ }^{10}$ Istituto Nazionale di Fisica Nucleare, Turin, Italy; ${ }^{11}$ Niels Bohr Institute, University of Copenhagen, DK2100 Copenhagen, Denmark; ${ }^{12}$ Istituto Nazionale di Fisica Nucleare, Bari, Italy; ${ }^{13}$ Universita' di Catania, Catania Italy; ${ }^{14}$ RIKEN, Wako, Japan; ${ }^{15}$ Istituto Nazionale di Fisica Nucleare, Section of Roma Tor Vergata, Italy; ${ }^{16}$ Joint Laboratory of Optics, Faculty of Science, Palacký University, Olomouc, Czech Republic; ${ }^{17}$ The University of Chicago, Chicago, IL, USA; ${ }^{18}$ Karlsruhe Institute of Technology, Karlsruhe, Germany; ${ }^{19}$ University of Wisconsin, Madison, WI, USA; ${ }^{20}$ Colorado School of Mines, Golden, CO, USA; ${ }^{21}$ Department of Astronomy, University of Maryland, College Park, MD, USA; ${ }^{22}$ NASA Goddard Space Flight Center, Greenbelt, MD, USA; ${ }^{23}$ Space Sciences Laboratory, University of California, Berkeley, CA, USA; ${ }^{24}$ Skobeltsyn Institute of Nuclear Physics, Lomonosov Moscow State University, Moscow, Russia; ${ }^{25}$ Center for Space Science \& Technology, University of Maryland, Baltimore County, Baltimore, MD, USA; ${ }^{26}$ Institute of Experimental Physics, Slovak Academy of Sciences, Kosice, Slovakia; ${ }^{27}$ Institute of Physics of the Czech Academy of Sciences, Prague, Czech Republic; ${ }^{28}$ Instituto de Ciencias Nucleares, UNAM, CDMX, Mexico; ${ }^{29}$ University of Iowa, Iowa City, IA, USA; ${ }^{30}$ University of Geneva, Geneva, Switzerland; ${ }^{31}$ Institutt for fysikk, NTNU, Trondheim, Norway; ${ }^{32}$ Istituto Nazionale di Fisica Nucleare, Napoli, Italy; ${ }^{33}$ Université de Paris, CNRS, Astroparticule et Cosmologie, F-75013 Paris, France; ${ }^{34}$ Universita di Roma Tor Vergata, Italy; ${ }^{35}$ Faculty of Physics, University of Warsaw, Warsaw, Poland; ${ }^{36}$ Istituto Nazionale di Fisica Nucleare - Laboratori Nazionali di Frascati, Frascati, Italy; ${ }^{37}$ Universita' di Napoli Federico II, Napoli, Italy; ${ }^{38}$ National Centre for Nuclear Research, Lodz, Poland; ${ }^{39}$ Fairfield University, Fairfield, CT, USA. 